\title{
Diabetes insipidus, diabetes mellitus, optic atrophy and deafness. A clinical and genetic study
}

\author{
N. A. NAGI \\ M.B., Ch.B. \\ College of Medicine, University of Mosul, Mosul, Iraq
}

\begin{abstract}
Summary
Two Iraqi sisters and a female cousin developed diabetes insipidus (DI), diabetes mellitus (DM), optic atrophy (OA), and deafness (D), (the 'DIDMOAD' syndrome) before the age of 12 years. One girl exhibited all the features of this disease complex only 3 months after an unusually late onset of recognizable symptoms at 11 years 9 months. Another girl died suddenly and unexpectedly. This family study illustrates the recessive inheritance pattern of the syndrome.
\end{abstract}

\section{Introduction}

Insulin-dependent childhood diabetes mellitus is occasionally associated with other serious disorders. These include diabetes insipidus, optic atrophy, deafness, endocrine abnormalities, and both central and autonomic neurological abnormalities. The development of diabetes mellitus and optic atrophy in the same patient was noted as long ago as 1871 by Allbutt. However, the cluster of disorders known as the DIDMOAD syndrome has become more commonly recognized since the report by Wolfram and Wagner (1938) of 4 siblings with diabetes mellitus and optic atrophy who later developed hearing loss and bladder dilatation.

The major interest of this syndrome lies in the variability of its expression, its pattern of inheritance, the pathogenesis of the different disorders and the eventual outcome. The following 3 case reports highlight some of these features.

\section{Case reports}

The 3 Iraqi children described were the offspring of consanguineous marriages of male first cousins both of whom married each other's sister (see family tree).

\section{Case 1}

A 4-year-old girl developed polyuria, polydipsia and enuresis of gradually increasing severity. The birth history and early development were normal apart from the consanguinity. At 5.5 years she was found to have vasopressin-sensitive diabetes insipidus. She had a normal glucose tolerance test (GTT), normal visual acuity with normal fundi and there was no subjective hearing loss. Vasopressin treatment was not available at the local clinic and at 7 years she was readmitted to hospital still passing 7-10 litres of urine per day with specific gravity (SG) consistently less than 1004. The urine now showed heavy glycosuria and a second GTT confirmed diabetes mellitus. Water deprivation over $9 \mathrm{hr}$ was associated with a weight loss of $1.5 \mathrm{~kg}$, continuing excessive urine excretion of low SG and a rise in plasma sodium. Hypertonic saline infusion failed to reduce the urine output but vasopressin (Pitressin) 3 units i.m. reduced the output from 7-10 litres to 2 litres/24 hr with an increase in SG from 1004 to 1016. Haemoglobin, blood urea, serum calcium, skull X-ray, air encephalogram and IVP were all normal. She had normal fundi and visual acuity; she had no subjective hearing loss.

At 9 years she was receiving lente insulin regularly but only occasional doses of vasopressin. A repeat IVP showed bilateral hydronephrosis and hydroureter (Fig. 2). Examination of the fundi now showed primary optic atrophy.

Despite strict instructions on treatment with both insulin and vasopressin, she died suddenly, 2 months after leaving hospital, with overt bleeding from the mouth, nose, urethra and vagina.

\section{Case 2}

A 5-year-old sister of case 1 developed polyuria, polydipsia and weight loss. The urine SG varied from 1015 to 1025 and contained excess glucose. Diabetes mellitus was confirmed by GTT. Treatment was started with lente insulin. The fundi were normal and there was no subjective hearing loss.

At 7 years she developed further polyuria in spite of insulin. Investigation confirmed a urine output of 3-4 litres/24 hr, SG $1005-1010$ rising to 1015 after 9 -hr water deprivation. Vasopressin 3 units i.m. reduced urine output to 1 litre/24 hr with a maximum 


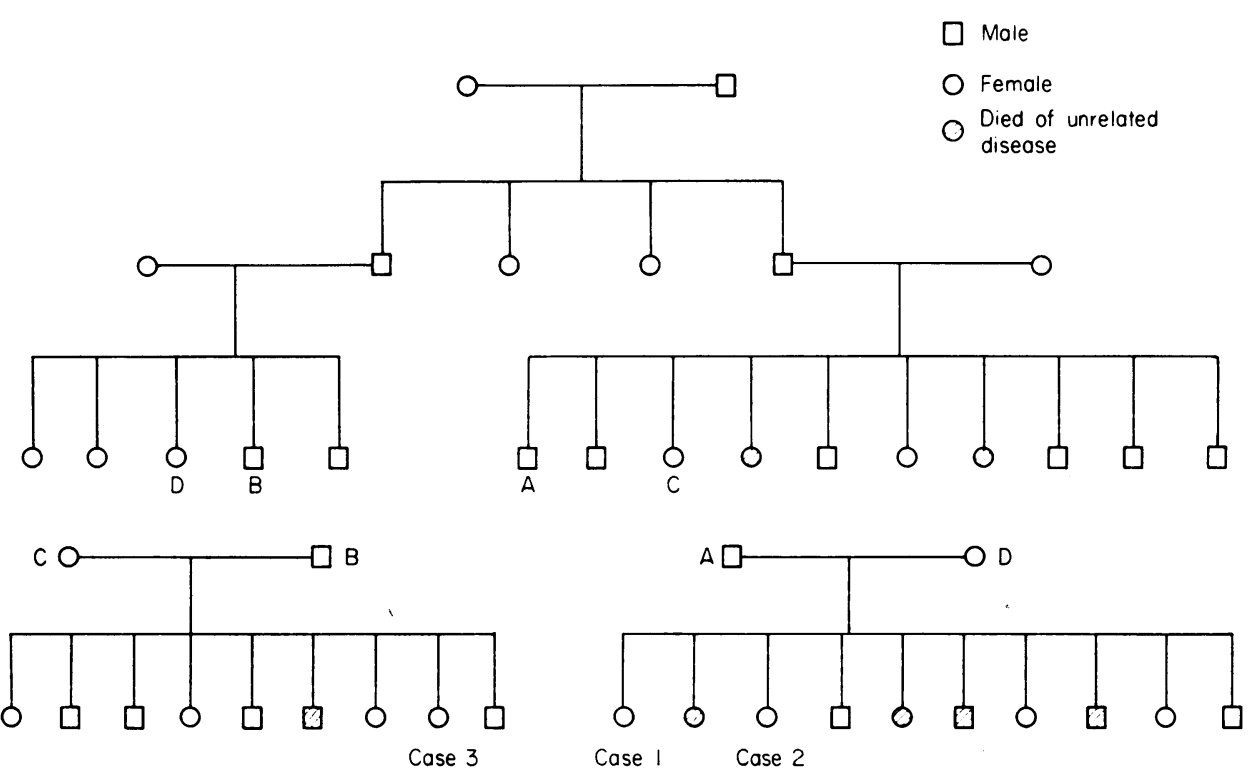

FIG. 1. Family tree showing recessive inheritance pattern of DIDMOAD syndrome.

SG of 1030. The IVP showed asymmetrical hydronephrosis and hydro-ureter, being more marked on the left (Fig. 3). An audiogram revealed bilateral sensori-neural hearing loss particularly of high tone frequencies. The pupils reacted poorly to light, bilateral optic atrophy was present and visual acuity was reduced $(R, 6 / 36 ; L, 6 / 60)$. Haemoglobin, blood urea, serum calcium, skull X-ray and EEG were normal.

\section{Case 3}

At the age of 11 years 9 months the female cousin of the 2 previous cases developed polyuria, polydipsia and weight loss. When examined 3 months after the onset of symptoms she had sluggishly reacting pupils, bilateral primary optic atrophy and visual acuity of $6 / 12$ in both eyes. An audiogram showed bilateral sensori-neural hearing loss mainly of high tone frequencies. The urine output was 3-4 litres/24 hr and despite heavy glycosuria the SG was below 1010. Water deprivation for $9 \mathrm{hr}$ caused a rise in SG to 1016. Vasopressin administration allowed a substantial decrease in urine output with a maximum SG of 1030. A GTT confirmed diabetes mellitus. Haemoglobin, blood urea, serum calcium, IVP, skull X-ray and EEG were all normal.

\section{Discussion}

These 3 children illustrate the variable timing of onset of the different disorders which form the disease complex known as the DIDMOAD syn- drome. Usually diabetes mellitus is the first disordero $\vec{\varphi}$ to be diagnosed as in case 2. Marquardt and Loriaux (1974) found that 35 of 43 cases presented. with diabetes mellitus before the age of 10 years.

In contrast, diabetes insipidus often develops insidiously in the second decade after diabetes mellitus has been present for some years. Case reports, however, have described diabetes insipidus as the presenting disorder as in case 1 (Raiti, Plotkin and Newns, 1963). The diabetes insipidus is caused by a deficiency of circulating vasopressin (Richardson and Hamilton, 1977) and hence should be eminently treatable with vasopressin analogues.

Urinary tract dilatation has been associated with the syndrome (Raiti et al., 1963; Moore, 1971). Hydronephrosis, hydro-ureter and bladder dilatation are recognized complications of untreated diabetes insipidus (Carter and Goodman, 1963; Wheeler and Adelson, 1964) and may be due to physical distension of the urinary tract by the enormous fluid output (Ten Bensel and Peters, 1970). The distension is therefore acquired rather than congenital, as illustrated by the change in IVP in case 1 . With adequate treatment of the polyuria the dilatation may disappear (Raiti et al., 1963; Marquardt and Loriaux, 1974).

Optic atrophy is a serious untreatable component of the syndrome. It is primary, bilateral and usually progressive, in some cases ending in blindness. It may occasionally precede the onset of the endocrine disturbances (Marquardt and Loriaux, 1974) but 


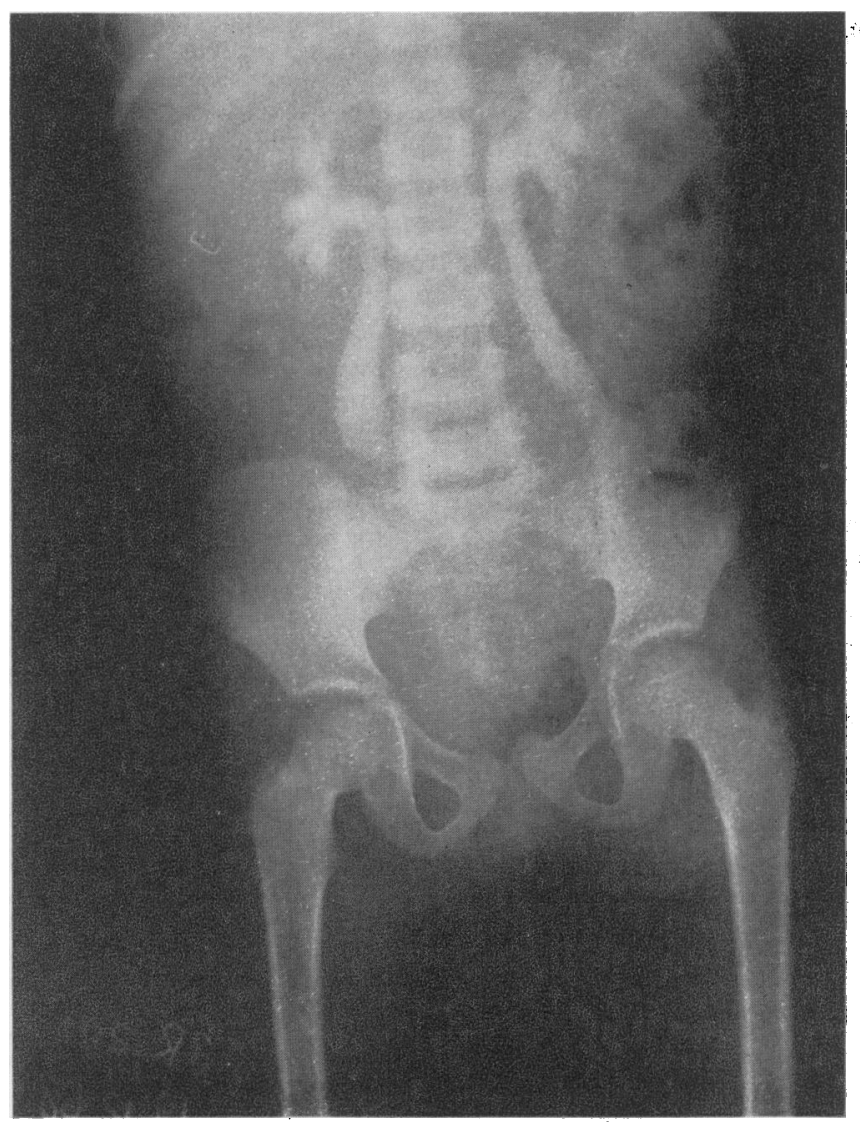

FIG. 2. Intravenous pyelography of case 1, showing bilateral hydronephrosis and bilateral hydro-ureter.

like diabetes insipidus is usually diagnosed in the second decade.

Most patients with this syndrome do not complain of subjective deafness (Wolfram and Wagener, 1938; Cooper et al., 1950) but audiographic study typically reveals bilateral high tone sensori-neural hearing loss. Fortunately, this usually remains mild and does not deteriorate rapidly although severe hearing deficits have been reported (Rose et al., 1966). The auditory problems usually arise after the age of 10 years although 2 cases became deaf before developing diabetes mellitus (Shaw and Duncan, 1958).

The third case was remarkable in displaying the fully developed syndrome at her first medical examination apparently only 3 months after the onset of symptoms. She is also unusual in that the onset was after the age of 10 years which is apparently later than in other reported cases.
Diabetes insipidus, optic atrophy and sensorineural deafness are frequently all neurogenic in origin and it is of interest that some patients have other neurological problems such as nystagmus, ataxia, epilepsy, abnormal EEGs and mental retardation (Rose et al., 1966). Autonomic or hypothalamic dysfunction manifesting as a loss of central temperature control mechanisms has also been described (Marquardt and Loriaux, 1974). Diabetes mellitus would normally be considered an endocrine disease of non-neurological, extracranial origin, but in this syndrome other endocrine abnormalities have been described such as infantilism, testicular atrophy with gynaecomastia (Wolfram and Wagener, 1938) and amenorrhoea (Ikkos et al., 1970) all of which might have had an intra-cranial hypothalamic origin. It is interesting to speculate whether diabetes mellitus in this constellation of disorders could also have a neurogenic origin. 


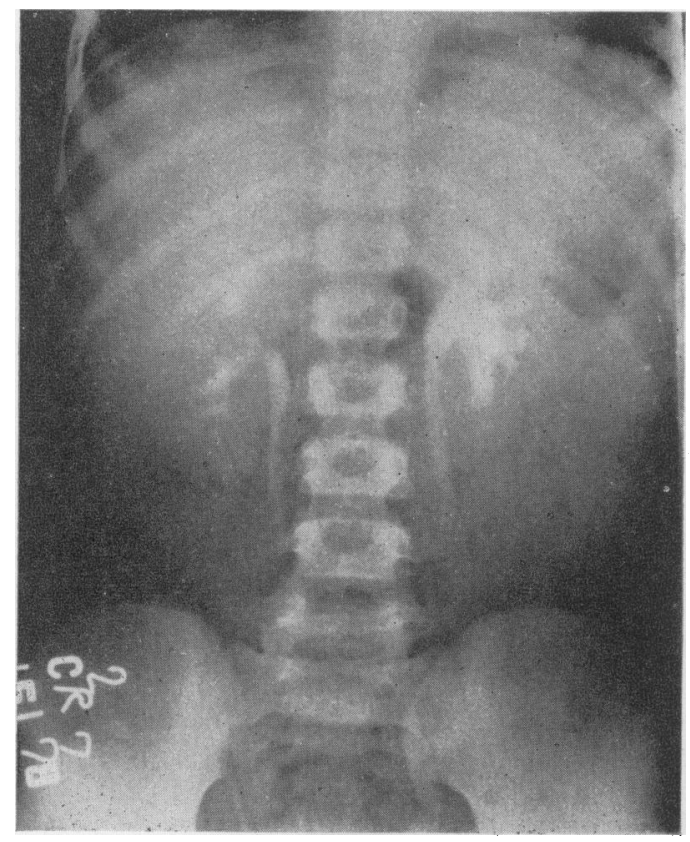

FIG. 3. Intravenous pyelography of case 2 showing hydronephrosis and hydro-ureter, more marked on the left side.

The DIDMOAD syndrome has been reported only in single generations except in the family described by Shaw and Duncan (1958) in 2 generations. Several authors have considered that the syndrome represents a single genetic trait, transmitted in Mendelian recessive manner (McKusick, 1971). The cases reported here were the products of unusual double consanguineous marriages and the fact that both marriages produced affected children further supports a recessive inheritance pattern. Carriers are undetectable at present: genetic counselling may help to avoid intermarriage which is still common in some countries.

It is difficult to be sure of the prognosis in a syndrome with variable manifestations, but a patient reported by Page, Asmal and Edwards (1976) died suddenly as did case 1 in this report. This raises the possibility that more extensive hypothalamicpituitary disturbances may develop during the natural history of the DIDMOAD syndrome

\section{Acknowledgments}

I am grateful to Dr P. G. F. Swift, consultant paediatrician at Leicester General Hospital, Leicester, England, for his guidance, critical evaluation and editorial help during the preparation of this paper.

Thanks are also extended to Drs S. Matloob and A. A. Quassim.

\section{References}

Allbutt, T.C. (1871) On the Use of the Ophthalmoscope in Diseases of the Nervous System and of the Kidneys, Also in Certain other General Disorders. MacMillan \& Co.. London \& New York.

CARTER, R.D. \& Goodman, A.D. (1963) Nephrogenic diabetes insipidus accompanied by massive dilatation of the kidneys, ureters and bladder. Journal of Urology, 89, 366.

CoOper, I.S., RYNearson, E.H., Bailey, A.A. \& MaCCARTY. C.S. (1950) The relation of spinal cord disease to gynecomastia and testicular atrophy. Proceedings. Mayo Clinic, 25, 320.

Ikkos, D.G., Fraser, G.R., Matsouki-Gavra, E. \& Petrochilos, M. (1970) Association of juvenile diabetes mellitus, primary optic atrophy and perceptive hearing loss in three sibs, with additional idiopathic diabetes mellitus insipidus in one case. Acta endocrinologica, 65, 95.

MARQUARDT, J.L. \& LoRIAUX, L. (1974) Diabetes mellitus and optic atrophy. Archives of Internal Medicine, 134, 32.

Moore, J.R. (1971) Juvenile diabetes mellitus, diabetes insipidus and neurological abnormalities, Proceedings of the Royal Society of Medicine, 64, 730.

MCKusicK, V.A. (1971) Mendelian Inheritance in Man. 3rd edn, p. 366. Johns Hopkins Press, Baltimore \& London.

Page, M. McB., Asmal, A.C. \& Edwards, C.R.W. (197ळ The syndrome of diabetes insipidus, diabetes mellitus optic atrophy and deafness. Quarterly Journal of Medicin 45, 505.

Raiti, S., Plotkin, S. \& Newns, G.H. (1963) Diabetes mellitus and insipidus in two sisters. British Medical Journal, 2, 1625.

Richardson, J.E. \& HamiLton, W. (1977) Diabetes insipidus, diabetes mellitus, optic atrophy and deafness: 3 cases of 'DIDMOAD' syndrome. Archives of Disease in Childhood, 52, 796.

Rose, F.C., Fraser, G.R., Friedman, A.I. \& Kohner, E.M. (1966) The association of juvenile diabetes mellitus and optic atrophy; clinical and genetical aspects. Quarterly Journal of Medicine, 35, 385.

Shaw, D.A. \& DunCan, L.J. (1958) Optic atrophy and nerve deafness in diabetes mellitus. Journal of Neurology, Neurosurgery and Psychiatry, 21, 47.

Ten Bensel, R.W. \& Peters, E.R. (1970) Progressive hydronephrosis, hydroureter and dilatation of the bladder in siblings with congenital nephrogenic diabetes insipidus. Journal of Pediatrics, 77, 439.

Wheeler, J.S. \& Adelson, W.J. (1964) Pituitary diabetes indipidus associated with progressive urinary tract dilatation. Journal of Urology, 92, 64.

WolfRAM, D.J. \& WAGENER, H.P. (1938) Diabetes mellitus and simple optic atrophy among siblings. Proceedings. Mayo Clinic. 13. 715. 